\title{
Innovación pública abierta Open innovation in the public sector
}

\author{
Julián Villodre \\ Universidad Autónoma de Madrid \\ ORCID ID 0000-0003-0468-8802 \\ julian.villodrede@uam.es
}

Cita recomendada:

Villodre, J. (2019). Innovación pública abierta. Eunomía. Revista en Cultura de la Legalidad, 17, 314-327.

doi: https://doi.org/10.20318/eunomia.2019.5036

Recibido / received: 03/06/2019

Aceptado / accepted: 28/07/2019

\begin{abstract}
Resumen
La innovación pública abierta es un concepto amplio, que habitualmente hace referencia al aprovechamiento de conocimiento interno y externo con el objetivo de transformar las organizaciones y generar valor público. Esta voz se centra en estudiar el concepto de innovación pública abierta explorando los orígenes y evolución del mismo, así como su relación con el paradigma de la gobernanza pública inteligente. Adicionalmente, la voz presenta las comunidades digitales de innovación y los laboratorios de innovación pública como aplicaciones prácticas del concepto.
\end{abstract}

\section{Palabras clave}

Innovación pública, laboratorios de innovación, innovación abierta, comunidades digitales de innovación, gobierno abierto, gobernanza pública inteligente.

\begin{abstract}
Open innovation in the public sector is a broad concept which usually refers to the use of internal and external knowledge with the aim of transforming organizations and generating public value. This paper focuses on studying the application of the open innovation concept to the public sector. For this purpose, the paper explores the origins and evolution of the concept, and its relationship with the Smart Governance paradigm. In addition, this paper presents digital innovation communities and living labs as practical applications of the concept.
\end{abstract}

\section{Keywords}

Public innovation, living labs, open innovation, digital innovation communities, open government, smart governance.

SUMARIO. 1. Introducción: la innovación pública abierta como concepto en el sector público. - 2. Origen y desarrollo de la innovación pública abierta. La innovación abierta y la gobernanza pública inteligente. - 3. Poniendo en marcha la innovación: las comunidades digitales de innovación y los laboratorios de innovación pública - 4 . Conclusiones. 
1. Introducción: la innovación pública abierta como concepto en el sector público

Las sociedades experimentan, cada vez más, una creciente complejidad de los problemas públicos. Los desafíos de las nuevas tecnologías (Bertot, Jaeger y Grimes, 2012), el progresivo abandono de la consideración del ciudadano como mero usuario de servicios (Hilgers y Ihl, 2010; Pollitt y Bouckaert, 2004) o la presión de agentes externos capaces de mantener el pulso ante unos Estado-nación cada vez más debilitados (Castells, 2009), entre otros, urgen al sector público a introducir reformas de calado en sus procesos, en las formas de gestionar los recursos y en el enfoque hacia la solución de los problemas. La innovación cerrada (Chesbrough, 2003) comienza a dar poco a poco paso a soluciones más abiertas capaces de enfrentarse a entornos complejos, a la aparición de redes ciudadanas e intermediarios digitales, o a las nuevas habilidades y la morfología de unos empleados públicos más comprometidos con dar protagonismo al ciudadano (Lee, Hwang y Choi, 2012). A lo largo de esta voz, nos centramos en definir el concepto de innovación pública abierta, estudiar sus orígenes, su evolución y relación con los paradigmas de gestión pública. Finalmente, observamos su realización práctica a través de las comunidades digitales de innovación y de los laboratorios de innovación pública.

Referirnos a innovación en el sector público suele ser sinónimo de introducir nuevos aspectos. Así, Green, Howells y Miles (2001) o Cunningham (2005) han tendido a definir la innovación pública como la creación e implementación de nuevos procesos, productos o servicios con el resultado de un incremento en la eficiencia, la eficacia o la calidad de los mismos. La propia Organización para la Cooperación y el Desarrollo Económicos (OCDE), ha calificado el concepto como la puesta en marcha de nuevos enfoques diseñados para obtener mayores resultados públicos en términos de eficiencia, eficacia y satisfacción ciudadana. Por otro lado, autores como Sánchez, Lasagna y Marcet (2013) ponen también el acento en la novedad como impulsor de la eficiencia y la eficacia, pero también, como proyección de ideas "realizables" para generar cambios que generen valor público, esto es, valor para los servicios del interés general (Meynhardt, 2009).

Sin embargo, la innovación no siempre tiene porque ser algo nuevo. Así, Pollit (2011) ha destacado que las administraciones siempre han estado adaptándose y regulando a través de procesos en los que no han sido pioneros. Sin embargo, el hecho de que las administraciones se apropien de cosas que tal vez no sean nuevas forma, en realidad, parte también de un proceso innovador. Autores como Rogers (2003), han llegado a delimitar las fases de introducción de cualquier innovación: desde los propios innovadores, creadores del nuevo proceso, producto, o servicio, hasta los más rezagados, forman parte del proceso de difusión de la innovación (Rogers, 2003). De hecho, y especialmente en relación a la introducción de tecnologías sociales (web 2.0) en el sector público, las innovaciones no suelen ser novedosas, pues por el carácter disruptivo de estas tecnologías, muchas administraciones prefieren esperar a que hayan sido probadas primero por otras organizaciones.

Esta disparidad de enfoques acentúa que no hay consenso claro sobre lo que debe o no debe ser considerado como innovación pública. El concepto de innovación pública es habitualmente amplio, y habla, entre otros, de productos, servicios, posicionamiento, provisión, estrategias, gobernanza y, en ocasiones, incluso es solo pura retórica (Pollit, 2011). Es un concepto "vital" para entender la transformación del sector público hoy en día, pero muy difícil de operacionalizar para analizarlo empíricamente (Pollit, 2011). Por otro lado, el concepto en muchas de sus 
definiciones ha sido vinculado a una manera concreta de entender la gestión pública, el paradigma de la Nueva Gestión Pública. En este paradigma, el papel de la innovación ha pasado habitualmente por mejorar la gestión operativa y estratégica a través de un incremento en la autonomía y flexibilidad del gestor y una orientación hacia la satisfacción del consumidor (Pettigrew, Whittington y Thomas, 2006).

La innovación abierta en el sector público (que en este artículo denominamos bajo el nombre de innovación pública abierta para reforzar su sustantividad, siguiendo corrientes propuestas por los propios empleados públicos ${ }^{1}$ ) tampoco cuenta con una definición teórica clara. Una de las definiciones clásicas del concepto de innovación abierta ha sido la dada por Chesbrough (2003), que entiende la innovación abierta como un modelo de gestión de la innovación en el cual las organizaciones abren sus procesos de transformación hacia fuera, combinando ideas internas y externas para generar valor añadido en sus servicios o productos. Esta definición, sin embargo, no fue creada teniendo en mente al sector público, aunque ha servido de inspiración para las aproximaciones de la innovación pública abierta (Bakici, Almirall y Wareham, 2013). Así, la innovación pública abierta ha tendido a definirse a través de su conexión e integración con actores externos a la administración pública, copartícipes de la creación de valor público (Hilgers y lhl, 2010), siendo denominado como citizensourcing cuando el proceso innovador pone al ciudadano en el foco de la idea y la transformación, como colaboradora de ésta junto a la administración (Mergel y Desouza, 2013; Hilgers y lhl, 2010).

La mayor parte de las definiciones sobre innovación pública abierta ponen el acento en la colaboración a través de plataformas digitales. Como comentan Mergel y Desouza (2013) y Mergel (2014), los procesos colaborativos de la innovación abierta ocurren habitualmente en plataformas digitales, a través de tecnologías directamente relacionadas con la web 2.0, y donde es conveniente destacar redes sociales especializadas en crowdsourcing (Challenge.gov, Github, DecideMadrid...), plataformas wikis para el conocimiento colaborativo, habitualmente etiquetadas bajo el concepto de WikiGovernment (Criado y Villodre, 2016; Noveck, 2009), e incluso comunidades digitales especializadas en innovar (por ejemplo, GovLoop, en EEUU, y NovaGob, en Iberoamérica). A pesar de esto, son todavía pocos los estudios que han investigado estas plataformas en profundidad, especialmente en relación con los resultados de las interacciones que ahí se producen (Schmidthuber et al., 2018). En esta voz, revisaremos algunas de estas comunidades digitales de innovación.

Sin embargo, la realidad del concepto de la innovación pública abierta no pasa únicamente por la colaboración digital. Prueba de ello ha sido la reciente aparición de los laboratorios de innovación pública, denominados también a través de sus palabras anglosajonas living labs o innovation labs. Estos se configuran como espacios presenciales de colaboración entre administraciones y actores externos, capaces de generar soluciones a nuevos "problemas malditos" (Sørensen y Torfing, 2012) a través de la experimentación y la colaboración (Criado, RojasMartín y Silván, 2017). Los living labs se han convertido en intermediarios de la innovación pública (Gascó, 2017), donde la innovación se produce habitualmente a través de dos ideas (Gascó, 2017; Almirall, Lee y Wareham, 2012): a) impulsar la coproducción de los ciudadanos y su participación en procesos de creación de nuevas soluciones o implementación de soluciones existentes en términos de

\footnotetext{
1 Véase para un mayor detalle el documento colaborativo online editado entre la Diputación de Castellón, la Red de Entidades locales por la Transparencia y Participación ciudadana de la FEMP y NovaGob.Lab, titulado "Innovación Pública Abierta: ideas, herramientas y valores para participar en la mejora de la administración", disponible en este enlace: https://novagob.org/documentos/innovacionpublica-abierta-ideas-herramientas-y-valores-para-participar-en-la-mejora-de-la-administracion
} 
igualdad con el resto de agentes y organizaciones y b) alcanzar el proceso innovador mediante la aplicación de metodologías específicas, basadas en dinámicas de construcción de comunidad o en experimentación en la vida real. En esta voz, trabajaremos también con los laboratorios de innovación, como forma de implementación de innovación abierta en el sector público.

2. Origen y desarrollo de la innovación pública abierta. La innovación abierta y la gobernanza pública inteligente

La innovación abierta nace como tal en las organizaciones privadas. Durante mucho tiempo, las organizaciones habían ocultado celosamente sus procesos de desarrollo de productos, y cualquier nueva idea sobre los mismos se trabajaba de forma "interna", como una actividad cerrada (Chesbrough, 2003). Esto era así porque confiaban en que el modelo funcionase en torno a un círculo supuestamente virtuoso: las organizaciones invierten recursos en sus departamentos de innovación y desarrollo, lo que lleva a la organización a realizar ciertos descubrimientos que, en última instancia, le permiten mejorar productos y servicios ampliando las ganancias de las ventas, re-invirtiendo parte de éstas nuevamente en desarrollo e innovación interna, y protegiéndolas a través de la propiedad intelectual (Chesbrough, 2006). En el sector público, la innovación cerrada ha tendido a construirse a partir de un oxímoron (Borins, 2002): las administraciones públicas se han visto habitualmente como monopolios sin competencia, por lo que no tienen incentivos para innovar, ni tampoco para buscar innovación fuera de sus estructuras, habitualmente dotadas de gran estabilidad y consistencia. Y cuando se han producido innovaciones, el modelo ha navegado entre las estrategias "top-down", donde cualquier cambio específico se ha generado desde la propia institución mediante prescripciones, regulaciones o apoyo, y "bottom-up", donde los gobiernos han facilitado el desarrollo y difusión de una innovación siempre generada dentro de las redes de la propia administración (Borins, 2002).

Sin embargo, ese círculo virtuoso no es tan válido hoy en día. La apertura hacia una forma más abierta de innovar comenzó en organizaciones privadas pioneras, como Adidas o Lego, que han aplicado desde hace ya unos años modelos basados en la filosofía "Do it Yourself' (hazlo tú mismo), depositando en los consumidores parte del proceso de innovación (Hilgers y lhl, 2010). En ese sentido, estas compañías han reconocido que la colaboración con sus consumidores directos es una fuente de conocimiento que debe ser aprovechada, especialmente cuando la complejidad de las demandas del mercado aumenta, y la información del entorno es valiosa para la supervivencia y éxito de la organización (Lukensmeyer y Torres, 2008).

El sector público ha comenzado también a abrazar modelos abiertos de innovación. Una de las primeras agencias en aplicar un modelo de innovación pública abierta fue la NASA, que puso en marcha un programa de retos ciudadanos para buscar nuevas soluciones a los desafíos de la agencia (Mergel, 2014). La introducción de la innovación pública abierta ha venido de la mano del gobierno abierto, como un modelo que anima a poner en marcha mecanismos de colaboración ciudadana, reutilizando la información pública ya compartida por los gobiernos a través de portales de datos abiertos, y empleada, posteriormente, en intermediarios de la innovación (Bakici, Almirall y Wareham, 2013). La reutilización y apertura de datos públicos promueve la innovación, convirtiendo a las administraciones públicas de meras proveedoras de servicios en parte del proceso de intermediación. De esta forma, actúan como gestoras de plataformas, y en última 
instancia, como catalizadores de nuevas aplicaciones y servicios (Ramírez-Alujas, 2013), de la misma forma que permite a nuevos actores el acceso a materia prima (datos) para la solución de los problemas que les afectan.

De hecho, gobierno abierto, innovación pública abierta y plataformas digitales forman un curioso triángulo que se retroalimenta constantemente con base en las sinergias desarrolladas por la filosofía inherente a todos estos conceptos. A partir de la apertura de procesos, se apela de alguna forma a la idea de contribuir, distribuir y compartir la responsabilidad de la producción y prestación de servicios (RamírezAlujas, 2013). Estos, son valores básicos necesarios para que los procesos de innovación pública abierta puedan llegar a buen puerto. De la misma forma, el gobierno abierto siembra el campo idóneo para generar impulsos hacia una sociedad más abierta, donde la información esté distribuida de forma transparente y donde la generación de capital social sea básica para avanzar hacia una mayor calidad de vida (Criado, Ruvalcaba-Gómez y Valenzuela-Mendoza, 2018). Este proceso, de hecho, puede ser considerado como un factor habilitador de cambio también dentro de las culturas administrativas tradicionales. Finalmente, el gobierno abierto se ha visto revitalizado en gran medida por el auge de esas tecnologías, que como veremos son, a su vez, facilitadoras de procesos de innovación pública abierta. Esto, gracias a determinadas características intrínsecas de esas plataformas, como la eliminación de ciertas barreras de acceso (Forte y Lampe, 2013) o sus capacidades para garantizar visibilidad y reconocimiento, facilitar espacios de colaboración y hacer posibles procesos de participación gracias a la bidireccionalidad de las interacciones (Stamati, Papadopoulos y Anagnostopoulos, 2015).

Por otro lado, las principales diferencias en la aplicación de estas innovaciones entre organizaciones públicas y privadas no están claras. Para algunos, como Nambisan (2008), hay una similitud principal: todas ellas pretenden incorporar conocimiento externo al proceso de innovación. En última instancia, el mejor uso de ese conocimiento externo debe ir encaminado a incrementar el valor generado (Lee, Hwang y Choi, 2012). Es en ese valor donde surgen las principales diferencias, pues, en el caso del sector público, se trata de un beneficio público hacia el interés general (Lee, Hwang y Choi, 2012) a través de la mejora de servicios y no tanto de la elaboración de nuevos productos. Finalmente, como indican Cunningham y Kempling (2009), cualquier proceso de innovación exigirá en el sector público un mayor esfuerzo de negociación y resolución de disputas, esto es, una mayor necesidad de interactuar constantemente con los agentes implicados en los problemas y diseños.

Los tipos de innovación también se encuentran diferenciados. En una revisión sistemática de literatura recientemente publicada sobre innovación en el sector público, de Vries, Bekkers y Tummers (2016) clasificaron los tipos de innovación en seis: a) la innovación en el proceso, centrada en el incremento de la calidad y eficiencia de los procedimientos internos y externos; b) innovación administrativa, centrada en la incorporación de nuevas formas organizativas, nuevos métodos de gestión pública, y técnicas de trabajo; c) innovación tecnológica, esto es, el empleo de nuevas tecnologías para la prestación de servicios; d) innovación en el servicio o producto, responsable de la creación de productos o servicios realmente novedosos; e) innovación en la gobernanza, con el desarrollo de nuevos procesos para enfrentarse y gestionar los problemas públicos y, finalmente, f) la innovación conceptual, centrada en el diseño de nuevos paradigmas que permitan repensar los problemas públicos y sus soluciones. 
El proceso de innovación pública abierta suele efectuarse a través del modelo crowdsourcing. Como han planteado Criado y Villodre (2016), Mergel y Desouza (2013) y Linders (2012), entre otros, este modelo se basa en un aprovechamiento consciente de la inteligencia colectiva, esto es, del conocimiento de otros actores, para dar solución a problemas públicos complejos que, de otra forma, requerirían de grandes esfuerzos en recursos por parte de las administraciones. Este proceso consta de diversas partes (Brabham, 2013): a) la administración selecciona a un colectivo o comunidad, más o menos formado, pero diferenciado de los habituales clubes de expertos; b) la comunidad se pone conjuntamente a buscar solución al problema, pudiendo las administraciones públicas incentivar el proceso (por ejemplo, con premios); c) el resultado final supone un beneficio mutuo para la administración y para la comunidad que ha trabajado conjuntamente en co-producir la solución.

Finalmente, y en relación con los resultados de la innovación pública abierta, a menudo son menos destacados, y lo hacen a través de sus supuestas ventajas. Muchos autores han enfatizado, entre sus principales beneficios, una mayor consciencia de las administraciones sobre los problemas públicos, prácticas más eficaces en base a las experiencias directas con el problema de los ciudadanos, y un incremento de la confianza del ciudadano con la administración. Sin embargo, en muchas ocasiones, los posibles beneficios de la innovación pública abierta se ven bloqueados por las propias regulaciones estrictas de las administraciones, que basadas en modelos antiguos de contratación pública, requieren a las administraciones conocer de ante mano la solución al problema. En ocasiones, las limitaciones vienen de la evaluación de la calidad de la solución. En estas, los propios comités parecen más interesados en verificar si la solución fue generada por un conjunto de expertos, que en validar cualquier creación comunitaria (Mergel y Desouza, 2013). En cualquier caso, la implementación de los resultados de la innovación pública abierta requerirá de una mayor flexibilidad en los procedimientos administrativos.

\begin{tabular}{|c|c|c|c|}
\hline Dimensión & $\begin{array}{c}\text { Administración } \\
\text { Pública } \\
\text { Tradicional }\end{array}$ & $\begin{array}{l}\text { Nueva Gestión } \\
\text { Pública }\end{array}$ & $\begin{array}{c}\text { Gobernanza } \\
\text { Pública Inteligente }\end{array}$ \\
\hline Foco de atención & $\begin{array}{c}\text { Consejos sobre } \\
\text { políticas públicas }\end{array}$ & $\begin{array}{c}\text { Gestión operativa } \\
\text { y estratégica }\end{array}$ & $\begin{array}{c}\text { Aprovechar la } \\
\text { Inteligencia colectiva }\end{array}$ \\
\hline Forma organizativa & $\begin{array}{c}\text { Organización } \\
\text { vertical }\end{array}$ & $\begin{array}{c}\text { De alta } \\
\text { autonomía }\end{array}$ & Peer-to-peer \\
\hline $\begin{array}{l}\text { Presión para mejorar el } \\
\text { rendimiento }\end{array}$ & $\begin{array}{l}\text { Baja (actitud } \\
\text { monopolista) }\end{array}$ & $\begin{array}{c}\text { Elevada, impulso } \\
\text { de las TICs }\end{array}$ & $\begin{array}{c}\text { Elevada, ciudadanía } \\
2.0\end{array}$ \\
\hline $\begin{array}{l}\text { Orientación hacia el } \\
\text { ciudadano }\end{array}$ & Usuario & Consumidor & Coproductor \\
\hline Rol del gestor & Limitado & Flexible & $\begin{array}{l}\text { Detector de nichos } \\
\text { de valor público }\end{array}$ \\
\hline Orientación del cambio & Bajo & Alto & Alto \\
\hline
\end{tabular}

Este cambio hacia un modelo abierto de innovación en el sector público se ha visto reflejado a través de los diferentes paradigmas de gestión. Estos modelos científicos nos permiten representar con sencillez la realidad administrativa, de forma que podamos descomponer sus procesos en partes de más fácil estudio. La Tabla 1 da cuenta de las diferencias en la forma de innovar con respecto a los principales paradigmas en el sector público. Hasta los años 80 , la predominancia de 
una gestión basada en los ideales de la Administración pública tradicional dio lugar a una extensión del modelo de innovación basado en diversos aspectos (Pettigrew, Whittington y Thomas, 2006): a) las grandes organizaciones verticales imprimieron una visión de innovación cerrada, si bien orientada con un carácter universal; b) la capacidad innovadora, sin embargo, estaba limitada, en gran parte por la fuerte cultura procedimental, y el gestor se veía constantemente incentivado a tomar decisiones incrementales; c) adicionalmente, los incentivos para innovar estaban limitados por la actitud monopolista del sector (Borins, 2002), que apenas recibía presiones del entorno.

La Nueva Gestión Pública supuso un cambio radical, y centró sus esfuerzos en fomentar la innovación en el sector público. Puesto que la orientación del modelo de gestión era cercana a un modelo "de mercado" (Haque, 2007), la visión que la Nueva Gestión Pública imprimió sobre los procesos de innovación en el sector público fue de mayor apertura y flexibilidad para el gestor, a la vez que intensamente centrada en la mejora de la eficiencia y eficacia de los procesos (Bekkers, Edelenbos y Steijn, 2011). Esta visión ha llegado hasta nuestros días, y habitualmente ha sido la forma natural de aproximarse a la innovación pública.

Sin embargo, y fruto de la expansión de la filosofía web 2.0, basada en una concepción más colaborativa de la producción y prestación de servicios, nuevos modelos de gestión han comenzado a emerger. La innovación que presentaba la Nueva Gestión Pública estaba demasiado centrada en alcanzar mayores márgenes de eficiencia y eficacia, y olvidaba aspectos como el papel más protagonista que la ciudadanía ha comenzado a tener en la solución de problemas públicos. Como paradigma, la gobernanza pública inteligente (Criado, 2016; Noveck, 2015), coloca la innovación en un plano completamente abierto, al basar sus premisas de gestión en la importancia de promocionar procesos de colaboración y de innovación para tejer redes peer-to-peer (entre pares), capturando el conocimiento externo coproducido entre ciudadanía y administración (Linders, 2012).

La gobernanza pública inteligente destaca por sus rasgos centrados en el aprovechamiento de inteligencia colectiva para generar innovación. Como destacan Criado y Villodre (2018), este nuevo paradigma se basa en: a) el uso de plataformas digitales, tanto para la participación ciudadana como para la colaboración interna y externa dentro de una organización; b) un nuevo estilo de gestión, centrado en expertos innovadores especializados en detectar y crear valor público mediante el uso de conocimiento colaborativo externo; c) la definición del ciudadano como coproductor de servicios junto a la administración; d) un entorno dominado por la llamada "economía colaborativa" y, finalmente, e) un modelo de interacción social basado en una noción de "comunidad" (Criado, 2016; Luna-Reyes y Gil-García, 2014). El despliegue de todo esto suele efectuarse a través de "intermediarios" de la innovación (Bakici, Almirall y Wareham, 2013), que permiten a las administraciones públicas entrar en contacto con la inteligencia colectiva. En esta voz, y sin ánimo de excluir otros, exploramos las comunidades digitales de innovación y los laboratorios de innovación como plataformas de intermediación para la innovación pública abierta. 
3. Poniendo en marcha la innovación: las comunidades digitales de innovación y los laboratorios de innovación pública

Para alcanzar altos grados de innovación, las organizaciones más pioneras en el sector público colaboran habitualmente con intermediarios de la innovación. Los intermediarios de la innovación son espacios habitualmente externos a la organización, que proporcionan oportunidades de colaboración y búsqueda de solución a problemas públicos con la ayuda de la ciudadanía, de organizaciones privadas e incluso de otras organizaciones públicas. El primer objetivo de estos intermediarios es generar redes de trabajo colaborativo y, posteriormente, atraer todas las ideas innovadoras generadas en ese trabajo, filtrando las mejores y llevando su realización práctica a las instituciones. El segundo objetivo clave es la ejecución de proyectos, cuando así lo necesitan las instituciones públicas. Finalmente, los intermediarios suelen generar conversación, a través de eventos o discusiones virtuales o presenciales, fomentando la concienciación sobre un tema público concreto (Bakici, Almirall y Wareham, 2013).

Uno de esos intermediarios es precisamente las plataformas virtuales. Estas plataformas se caracterizan por su facilidad para potenciar la interacción entre administración y actores externos. Entre ellas debemos incluir tecnologías como las redes sociales generalistas (Facebook, Weiboo, etc.), plataformas de microblogging (Twitter), blogs, wikis, plataformas de medios para carga de imágenes y video (Instagram, Flickr, Youtube, etc.), y comunidades especializadas (Linkedln, o aquellas enfocadas en el sector público como GovLoop y NovaGob) e incluso mundos virtuales (Sansar, Second Life, Imvu, etc.). Estas plataformas se caracterizan, como señalan Forte y Lampe (2013), por el apoyo a la creación colectiva de un producto o servicio, presentando pocas barreras de entrada y salida, y bajo el soporte de estructuras caracterizadas por su alta persistencia y maleabilidad. Cuando el propósito de uso de algunas de estas plataformas digitales se encamina hacia la innovación pública, podemos denominarlas convenientemente como comunidades digitales de innovación pública (Criado y Villodre, 2018). A continuación, ofrecemos algunos ejemplos de estas comunidades digitales de innovación:

\section{- Challenge.gov (Figura 1)}

(https://www.challenge.gov/)

Esta plataforma fue lanzada por la Administración de Servicios Generales (GSA) del Gobierno de Estados Unidos en 2010. La plataforma respondía a una nueva directiva de política pública, bajo la denominación de "Prizes and Contests" (premios y concursos). La plataforma permite a las diferentes agencias públicas publicar en la red el problema o reto a resolver, y los ciudadanos interactúan en la plataforma en busca de una solución. Esas interacciones se desarrollan en un ambiente competitivo, que premia las mejores soluciones con algún incentivo o galardón. Los ciudadanos, además, pueden evaluar otras soluciones propuestas, votarlas e incluso involucrarse en la implementación de estas. Para conocer más sobre esta plataforma, recomendamos el análisis efectuado por Mergel y Desouza (2013) en Public Administration Review.

- Future of Melbourne Wiki (http://www.futuremelbourne.com.au/wiki/view/FMPlan)

Se trata de una wiki abierta al ciudadano de Melbourne, la ciudad con el crecimiento más rápido de Australia. Su lema, "the city plan that anyone can edit" (el plan de ciudad que cualquiera puede editar), alude directamente a la filosofía 
del WikiGovernment (Noveck, 2009), esta es, la de generar contenido colaborativo a través de sucesivas ediciones de documentos (denominados wiki). Para conocer más sobre esta plataforma u otras derivadas del gobierno wiki, recomendamos el análisis efectuado por Criado y Villodre (2016) publicado por el Instituto Nacional de Administración Pública (INAP).

\section{- NovaGob, la red social de la administración pública} (https://red.novagob.org/).

Se trata de una red social de ámbito iberoamericano centrada especialmente en los empleados públicos. NovaGob permite a empleados de diferentes administraciones públicas iberoamericanas entrar en contacto entre ellos, y con otros actores involucrados en el sector público, como organizaciones externas, consultores y académicos. Para conocer más, recomendamos el análisis de Criado y Villodre (2018), en la revista First Monday.

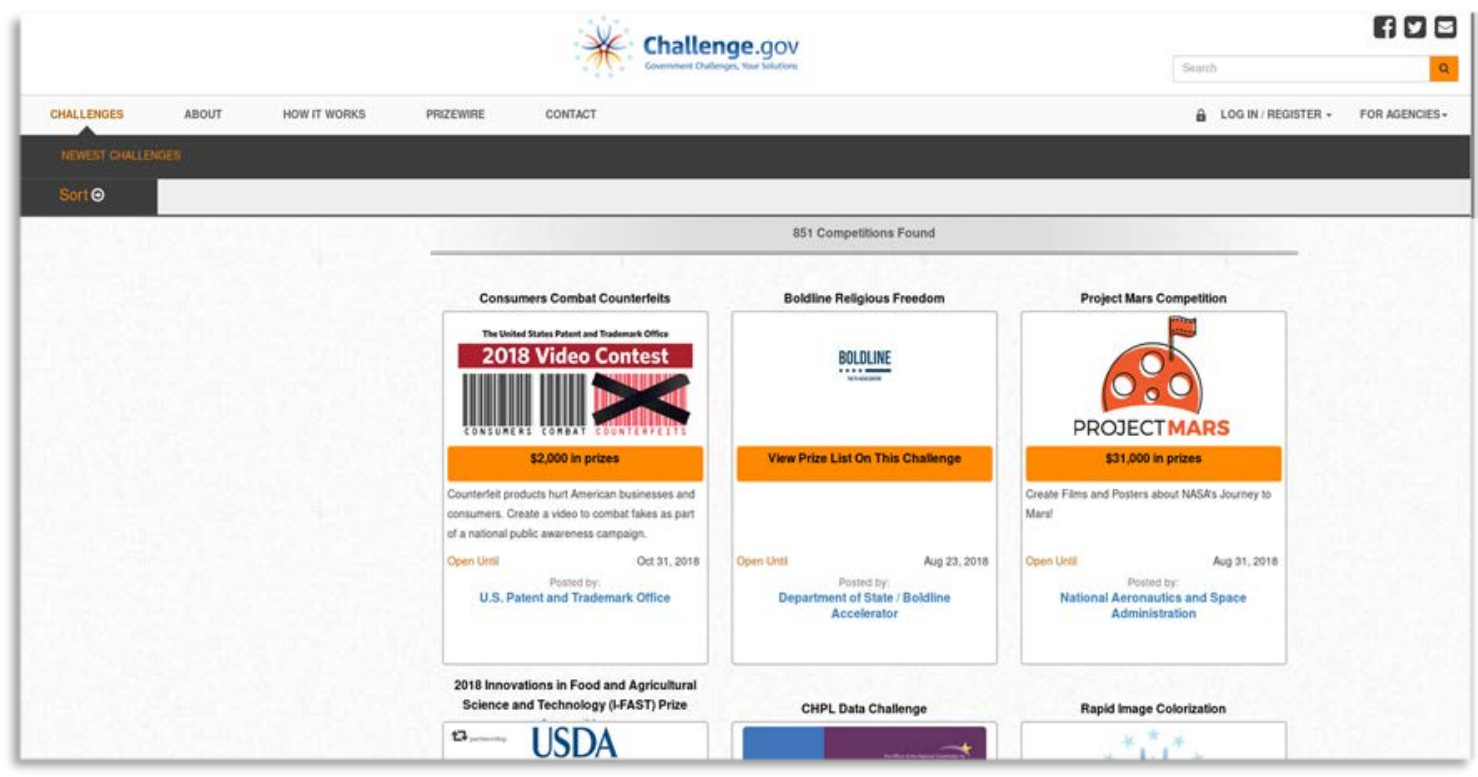

Figura 1. Página principal de Challenge.gov

Otro de esos posibles intermediarios para la innovación pública abierta son los laboratorios de innovación. También conocidos como living labs, se trata de espacios para la experimentación en las organizaciones públicas (Criado, RojasMartín y Silván, 2017). Tradicionalmente, los laboratorios de innovación eran espacios más cerrados, centrados en intercambios entre corporaciones privadas y públicas (Gascó, 2017), pero recientemente, impulsados por nuevos modelos de innovación, se han convertido en referentes en la mediación entre ciudadanos, organizaciones públicas y privadas, capturando y codificando las visiones ciudadanas en entornos experimentales reales (Gascó, 2017).

Al igual que las comunidades digitales de innovación, los laboratorios de innovación pública desarrollan el modelo abierto de innovación extrayendo conocimiento de las interacciones entre los diferentes agentes implicados. En general, los laboratorios de innovación cumplen tres funciones básicas (Gascó, 2017): a) conectan individuos con organizaciones; b) ofrecen espacios para el intercambio de ideas y conocimiento y c) proveen servicios tecnológicos, y modelos metodológicos, enfocados al aprendizaje continuo y la experimentación. A continuación, ofrecemos algunos ejemplos de laboratorios de innovación pública: 


\section{- Laboratorio de Gobierno de Chile} (https://lab.gob.cl)

Creado en 2014 por el Gobierno de Chile, este laboratorio se centra en el diseño de servicios públicos y la formación de empleados reorientando y concienciando en políticas públicas innovadoras. El laboratorio está representado por una red de colaboración ministerial, y su labor es especialmente destacada en el ámbito sanitario, a través de iniciativas como Impacta Salud (una convocatoria desarrollada el año 2015 con la idea de abrir el Estado a las ideas de diversos sectores para buscar en conjunto soluciones a la pregunta ¿cómo acercar la salud a las personas?).

\section{- The GovLab}

(http://thegovlab.org/)

Lanzado por la Universidad de Nueva York, The GovLab está muy centrada en la aplicación de innovación tecnológica en el sector público bajo el modelo de gobierno abierto. En The GovLab: a) se desarrollan y prueban prototipos para nuevas plataformas capaces de habilitar la colaboración e innovación pública abierta en instituciones públicas; b) diseminan información sobre los éxitos (y fracasos) de las innovaciones gubernamentales con el objetivo de aprender; c) entrenan a empleados públicos para poner en marcha proyectos abiertos de innovación en el sector público y d) generan, en última instancia, conexiones entre innovadores que les permita trabajar de forma colaborativa en marcos para una mejor gobernanza.

\section{- NovaGob Lab (Figura 2)}

(https://novagob.org/goblab)

Lanzado en 2016, y ubicado en la iniciativa de la red social NovaGob, el NovaGob Lab se acerca a The GovLab en su diseño híbrido experimental, así como en la utilización de prototipos y el ánimo de transferir conocimiento hacia el sector público y la sociedad. Puede encontrarse más información sobre este caso en el artículo de Criado, Rojas-Martín y Silván (2017), publicado en la revista Gestión Pública.

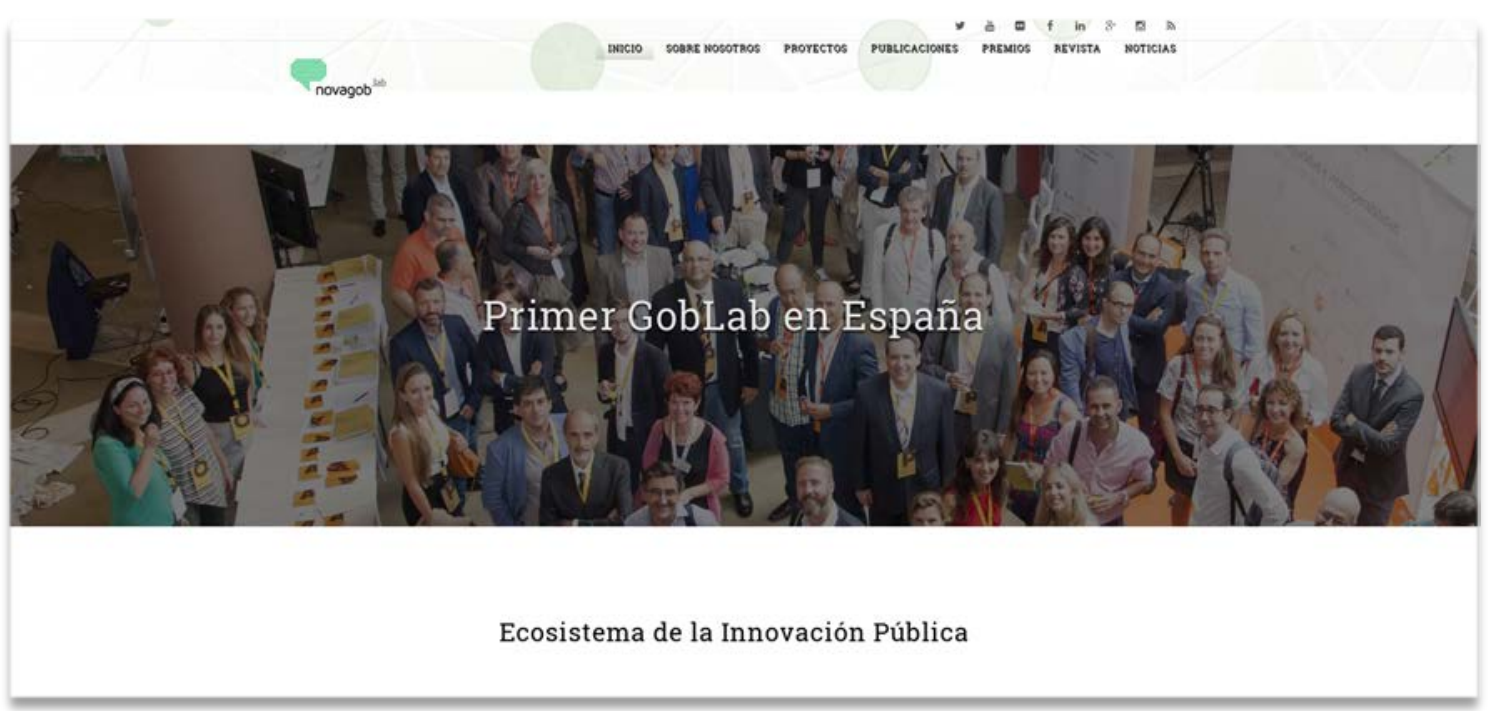

Figura 2. Página principal del NovaGob Lab. 


\section{Conclusiones}

Esta voz ha trabajado con el concepto de innovación pública abierta. A través de sus orígenes y desarrollo, la voz se ha aproximado a una nueva corriente que impulsa una innovación pública más abierta, y que se puede vincular con el desarrollo de nuevos paradigmas en el sector público relacionados con la colaboración, la coproducción y el aprovechamiento de la inteligencia colectiva, como la gobernanza pública inteligente. A través de dos intermediarios de innovación pública abierta, las comunidades digitales de innovación y los laboratorios de innovación pública, observamos cómo se desarrolla este fenómeno en el sector público. El concepto de innovación pública abierta se ha configurado, así, como un término diferencial en torno a varias características que podrían componer su definición: a) el aprovechamiento de inteligencia colectiva de actores externos (aunque, en ocasiones, también internos) del sector público mediante procesos peer-to-peer y crowdsourcing; b) el hecho de encontrarse cada vez más intermediada (aunque puede no estarlo) mediante plataformas digitales o espacios de experimentación y c) la presencia, como resultado, de productos dotados de gran valor público, reforzados y dependientes de la legitimidad de los actores involucrados, siendo ese resultado beneficioso tanto para la administración cómo para los actores, que en muchos casos, podrían ser los propios receptores de la innovación.

Aunque la voz ha intentado ofrecer una visión integral sobre un concepto emergente en la literatura sobre gestión pública, algunos aspectos deben ser considerados como limitaciones de este artículo. En primer lugar, esta voz se ha centrado en revisar el concepto de innovación pública abierta en relación con el paradigma de la gobernanza pública inteligente. Aunque la voz reconoce en varias ocasiones que este concepto bebe también del gobierno abierto (Ramírez-Alujas, 2013), futuros avances deberían incorporar una revisión más sosegada de la relación con ese concepto. Por otro lado, las realizaciones prácticas del concepto de innovación pública abierta se han estudiado fundamentalmente sobre la base de intermediarios de la innovación, por el cariz e importancia que están adquiriendo en los últimos años. Sin embargo, debe aclararse que la innovación pública abierta también puede darse sin necesidad de intermediarios y fuera de comunidades digitales y living labs. Finalmente, debemos entender que el término de innovación pública abierta es un término reciente, que va ganando sustantividad propia en base a la literatura existente sobre innovación pública e innovación abierta (Meijer, 2015). Continuar monitorizando la literatura reciente sobre innovación pública abierta será importante (Mergel, 2018): se trata de procesos que desafían las, todavía muy presentes, lógicas de innovación tradicionales en el sector público, altamente reguladas y con procesos de acción rígidos.

El interés por la innovación pública, y en especial, por los nuevos modelos de innovación abierta en el sector público va en aumento. Conforme el interés se mantenga en el tiempo, es muy probable observar un aumento en la literatura, en los marcos de análisis y en los estudios de caso, actualmente escasos en relación con la innovación pública abierta y sus nuevos intermediarios. Estos estudios tendrán, además, importantes implicaciones prácticas para el sector público, en tanto a que habilitarán nuevas perspectivas que podrán servir para un mejor enfoque de la innovación. En ese sentido, es importante que los gestores públicos no se dejen llevar por la fiebre de la innovación, como si ésta se tratase de un fin en sí mismo. Por un lado, y con especial atención a su dimensión tecnológica, por la posible persistencia de una brecha digital capaz de dinamitar la legitimidad de estos procesos. Por otro lado, y en relación a su propósito, la innovación pública abierta deberá siempre atender a un objetivo, siendo, en ese sentido, capaces de responder a la pregunta: innovar, ¿para qué? 


\section{Bibliografía}

Almirall, E., Lee, M., y Wareham, J. (2012). Mapping living labs in the landscape of innovation methodologies. Technology innovation management review, September 2012, pp. 12-18.

Bakici, T., Almirall, E., y Wareham, J. (2013). The role of public open innovation intermediaries in local government and the public sector. Technology Analysis \& Strategic Management, 25(3), pp. 311-327.

Bekkers, V., Edelenbos, J., y Steijn, B. (2011). Linking Innovation to the Public Sector: Contexts, Concepts and Challenges. En V. Bekkers, J. Edelenbos, J., y B. Steijn (eds.), Innovation in the public sector. Linking capacity and leadership (pp. 3-34). Nueva York, Estados Unidos: Palgrave MacMillan.

Bertot, J. C., Jaeger, P. T., y Hansen, D. (2012). The impact of policies on government social media usage: Issues, challenges, and recommendations. Government Information Quarterly, 29, pp. 30-40.

Borins, S. (2002). Leadership and innovation in the public sector. Leadership \& Organization Development Journal, 23 (8), pp. 467-476.

Brabham, D. C. (2013). Using Crowdsourcing in Government. IBM Center for the Business of Government (Collaborating across Boundaries). Recuperado de http://www.businessofgovernment.org/report/using-crowdsourcing-government [Última consulta: 30-07-2018]

Castells, M. (2009). Comunicación y Poder. Madrid, España: Alianza.

Chesbrough, H. (2006). Open innovation: The new imperative from creating and profiting from technology. Boston, Estados Unidos: Harvard Business School Press.

Chesbrough, H.W. (2003). Open innovation. A new paradigm for understanding industrial innovation. En H. Chesbrough, W. Vanhaverbeke y J. West (eds.) (2006), Open innovation. Researching a new paradigm (pp. 1-15). Oxford: Oxford University Press.

Criado, J. I., Ruvalcaba-Gomez, E. A. y Valenzuela-Mendoza, R. (2018). Revisiting the Open Government Phenomenon. A Meta-Analysis of the International Literature. JeDEM-eJournal of eDemocracy and Open Government, 10(1), pp. 50-81.

Criado, J. I., y Villodre, J. (2018). Public employees in social media communities: Exploring factors for internal collaboration using social network analysis. First Monday, 23 (4). Recuperado de https://journals.uic.edu/ojs/index.php/fm/article/view/8348/6661

Criado, J. I., Rojas-Martín, F., y Silván, A. (2017). Laboratorios de innovación para cambiar la gestión pública. Análisis del caso NovaGob Lab. Revista de Gestión Pública, 6 (1), pp. 19-42.

Criado, J. I. (2016). Gobernanza inteligente, innovación abierta y tecnologías sociales en unas administraciones públicas colaborativas. ¿Hacia un cambio de paradigma? En J.I. Criado (ed.) (2016), Nuevas tendencias en la gestión pública (pp. 27-52). Madrid, España: Instituto Nacional de Administración Pública.

Criado, J. I., y Villodre, J. (2016). Crowdsourcing y plataformas colaborativas en las administraciones públicas. ¿Hacia un WikiGovernment? En J.I. Criado (ed.) (2016), Nuevas tendencias en la gestión pública. Innovación abierta, gobernanza inteligente $y$ tecnologías sociales en unas administraciones públicas colaborativas (pp. 95-119). Madrid, España: INAP.

Cunningham, J. B., y Kempling, J.S. (2009). Implementing change in public sector organizations, Management Decision, 47 (2), pp. 330-44.

Cunningham, P. (2005). Innovation in the Public Health sector: A case study analysis. Informe publicado por NIFU Step Oslo. 
De Vries, H., Bekkers, V., y Tummers, L. (2016). Innovation in the public Sector: A Systematic Review and Future Research Agenda. Public Administration, 94 (1), pp. 146-166.

Forte, A., y Lampe, C. (2013). Defining, Understanding, and Supporting Open Collaboration Lessons from the Literature. American Behavioral Scientist, 57(5), pp. 535-547.

Gascó, M. (2017). Living Labs: Implementing Open Innovation in the Public Sector. Government Information Quarterly, 34(1), pp. 90-98.

Green L, Howells J, Miles I. (2001). Services and Innovation: Dynamics of Service Innovation in the European Union. Informe final PREST y CRIC University of Manchester.

Haque, S. (2007). Introduction: growing concerns for public accountability under the state in transition. Public Administration Quarterly, 31(4), pp. 386-96.

Hilgers, D., y IHL, C. (2010). Citizensourcing: Applying the Concept of Open Innovation to the Public Sector. The International Journal of Public Participation, 4(1), pp. 67-88.

Lee, S. M., Hwang, T., y Choi, D. (2012). Open innovation in the public sector of leading countries. Management Decision, 50(1), pp. 147-162.

Linders, D. (2012). From E-Government to We-Government: Defining a Typology for Citizen Coproduction in the Age of Social Media. Government Information Quarterly, 29, pp. 446-454.

Lukensmeyer, C. J., y Torres, L. H. (2008). Citizensourcing: Citizen Participation in a Networked Nation. En K. Yang y E. Bergrud (eds.) (2008), Civic Engagement in a Network Society (pp. 207-233). Charlotte, North Carolina: Information Age Publishing.

Luna-Reyes, L., y Gil-García, J. (2014). Digital government transformation and Internet portals: The co-evolution of institutions, technology, and organizations. Government Information Quarterly, 31(4), pp. 545-555.

Meijer, A. (2015). E-Governance Innovation: Barriers and Strategies. Government Information Quarterly, 32(2), pp. 198-206.

Mergel, I. (2018). Open innovation in the public sector: drivers and barriers for the adoption of Challenge.gov. Public Management Review, 20(5), pp. 726-745.

Mergel, I. (2014). Opening Government: Designing Open Innovation Processes to Collaborate With External Problem Solvers. Social Science Computer Review, pp. 1-15. Recuperado de https://www.cbs.dk/files/cbs.dk/mergel 2014 sscr.pdf

Mergel, I., Desouza, K. (2013). Implementing Open Innovation in the Public Sector: The Case of Challenge.gov. Public Administration Review, 73(6), pp. 882-890.

Meynhardt, T. (2009). Public Value Inside: What is Public Value Creation?. International Journal of Public Administration, 32(3-4), pp. 192-219.

Nambisan, S. (2008). Transforming Government through Collaborative Innovation. Informe IBM Centre for the Business of Government research report.

Noveck, B. S. (2015). Smart Citizens, Smarter State. Cambridge: Hardvard University Press.

Noveck, B. S. (2009). Wiki-government: How Open-source Technology Can Make Government Decision-making more Expert and more Democratic. Washington, DC, Estados Unidos: Brookings Institution Press.

Pettigrew, A., Whittington, R., y Thomas, H. (2006). Handbook of Strategy and Management. Londres: Sage.

Pollit, C. (2011). Innovation in the Public Sector: An Introductory Overview. En V. Bekkers, J. Edelenbos y B. Steijn (eds.) (2011), Innovation in the public sector. Linking capacity and leadership (pp. 35-43). Nueva York, Estados Unidos: Palgrave MacMillan.

Pollitt, C., y Bouckaert, G. (2004). Public Management Reform. Oxford: Oxford University Press. 
Ramírez-Alujas, A. (2013). Gobierno Abierto. Eunomía. Revista en Cultura de la Legalidad, 5, pp. 201-216.

Rogers, E. M. (2003). Diffusion of Innovations. New York, Estados Unidos: Free Press.

Sánchez, C., Lasagna, M., y Marcet, X. (2013). Innovación Pública: un modelo de aportación de valor. Santiago de Chile, Chile: RIL Editores.

Schmidthuber, L., y Hilgers, D. (2018). Unleashing Innovation beyond Organizational Boundaries: Exploring Citizensourcing Projects. International Journal of Public Administration, 41(4), pp. 268-283.

Sørensen, E., y Torfing, J. (2012). Collaborative Innovation in the Public Sector. The Innovation Journal: The Public Sector Innovation Journal, 17(1), pp. 1-14.

Stamati, T., Papadopoulos, T., y Anagnostopoulos, D. (2015). Social media for openness and accountability in the public sector: Cases in the Greek context. Government Information Quarterly, 32, pp. 12-29. 